\title{
Evolução fatal da co-infecção doença de Chagas/Aids: dificuldades diagnósticas entre a reagudização da miocardite e a miocardiopatia chagásica crônica
}

\author{
Fatal evolution of Chagas'disease/Aids co-infection: diagnostic difficulties \\ between myocarditis reactivation and chronic chagasic myocardiopathy
}

\author{
Eros Antonio de Almeida ${ }^{1}$, Eliane Lages Silva ${ }^{2}$, Maria Elena Guariento ${ }^{1}$, \\ Milton Lopes de Souza ${ }^{1}$, Francisco Hideo Aoki ${ }^{1}$ e Rogério de Jesus Pedro ${ }^{1}$
}

\begin{abstract}
RESUMO
A doença de Chagas é uma parasitose causada pelo protozoário Trypanosoma cruzi, transmitido por insetos triatomíneos. A doença ocorre desde o sul dos Estados Unidos da América do Norte até a Argentina, sendo que, aproximadamente, 14 milhões de pessoas devam estar infectados na América Latina, predominantemente na forma crônica da doença. A reagudização da doença de Chagas pode ocorrer em imunossuprimidos, como tem sido observado em pacientes com aids. Verificou-se descompensação cardíaca em um destes casos, com grave disfunção ventricular e arritmias sendo considerada a possibilidade de reagudização da doença de Chagas no miocárdio, uma vez que o xenodiagnóstico foi positivo. Face a gravidade foi tratado especificamente para o Trypanosoma cruzi com benznidazol, porém sem completar o tempo estipulado para este fim, vindo a falecer em consequiência de complicações da cardiopatia. A necropsia apresentou os estigmas habituais da cardiopatia chagásica crônica como miocardite fibrosante e redução do número de neurônios no tubo digestório, não sendo encontradas formas amastigotas do Trypanosoma cruzi em nenhum dos tecidos examinados. Assim, não ficou demonstrada a reagudização da doença de Chagas, mas sim evolução natural da cardiopatia chagásica crônica.
\end{abstract}

Palavras-chaves: Chagas. Tripanosomíase. Imunodeficiência adquirida. Reativação. Co-infecção.

\section{ABSTRACT}

Chagas disease is a type of parasitosis caused by the protozoan Trypanosoma cruzi, and it is transmitted by triatomine insects. This disease is found between the southern United States to Argentina and approximately 14 million people in Latin America are believed to be infected, predominantly with the chronic form of the disease. Reactivation of Chagas disease can occur among immunosuppressed patients, as has been observed among AIDS patients. In one such case, we observed cardiac decompensation with severe ventricular dysfunction and arrhythmias. This case was thought to be reactivation of Chagas disease in the myocardium, since the xenodiagnosis was positive. Specific treatment for Trypanosoma cruzi was administered, consisting of benznidazole, but the course of treatment was not completed because the patient died due to cardiopathic complications. The necropsy showed the usual stigmas of chronic Chagas cardiopathy, such as fibrosing myocarditis and a decreased number of neurons in the digestive system. There were no amastigote forms of Trypanosoma cruzi in any of the tissue samples studied. Therefore, reactivation of Chagas disease was not demonstrated but, rather, the natural evolution of chronic Chagas cardiopathy was demonstrated.

Key-words: Chagas. Trypanosomiasis. Acquired immunodeficiency. Reactivation. Coinfection.

A doença de Chagas ou tripanosomíase sul-americana é uma antropozoonose endêmica, ocorrendo desde o México até o sul da Argentina, causada por um protozoário flagelado, denominado Trypanosoma cruzi, transmitido ao homem e outros mamíferos por insetos hematófagos triatomínicos ${ }^{10}$. A forma de transmissão

1. Departamento de Clínica Médica, Faculdade de Ciências Médicas da Universidade Estadual de Campinas, Campinas, SP. 2. Disciplina de Parasitologia, Universidade Federal do Triângulo Mineiro, Uberaba, MG.

Endereço para correspondência: Dr. Eros Antonio de Almeida. Dept ${ }^{0}$ de Clínica Médica/FCM/UNICAMP. R. Alexander Fleming 40, Cidade Universitária Zeferino Vaz, 13083-970 Campinas, SP

Tel: 55 19 3521-7803; 5519 9257-9269; Fax: 55 19 3289-4107

e-mail: eros@fcm.unicamp.br

Recebido para publicação em 06/11/2008

Aceito em 20/03/2009 vetorial é a mais importante do ponto de vista de saúde pública, porém outras formas de transmissão existem, sendo a por transfusão sangüínea a segunda em importância epidemiológica. Após o contágio, ocorre uma fase aguda à qual se segue uma fase crônica, aproximadamente, depois de quatro meses da infecção e esta tem evolução variável em diferentes formas clínicas. É estimado que aproximadamente 14 milhões de pessoas estejam infectadas na América Latina e que 60 milhões estejam em risco de infecção, sendo que no Brasil, de 1,8 a 2,4 milhões de indivíduos devam estar na fase crônica da doença, 1/3 deles na forma cardíaca e digestiva, gerando alta morbi-mortalidade ${ }^{1}$.

Com o surgimento da infecção pelo vírus da imunodeficiência humana e o desenvolvimento da síndrome da imunodeficiência adquirida, passou a ser descrita a co-infecção desta com a doença de Chagas ${ }^{6} .0$ movimento migratório ocorrido no Brasil, 


\title{
Evolução fatal da co-infecção doença de Chagas/Aids: dificuldades diagnósticas entre a reagudização da miocardite e a miocardiopatia chagásica crônica
}

\author{
Fatal evolution of Chagas'disease/Aids co-infection: diagnostic difficulties \\ between myocarditis reactivation and chronic chagasic myocardiopathy
}

\author{
Eros Antonio de Almeida ${ }^{1}$, Eliane Lages Silva ${ }^{2}$, Maria Elena Guariento ${ }^{1}$, \\ Milton Lopes de Souza ${ }^{1}$, Francisco Hideo Aoki ${ }^{1}$ e Rogério de Jesus Pedro ${ }^{1}$
}

\begin{abstract}
RESUMO
A doença de Chagas é uma parasitose causada pelo protozoário Trypanosoma cruzi, transmitido por insetos triatomíneos. A doença ocorre desde o sul dos Estados Unidos da América do Norte até a Argentina, sendo que, aproximadamente, 14 milhões de pessoas devam estar infectados na América Latina, predominantemente na forma crônica da doença. A reagudização da doença de Chagas pode ocorrer em imunossuprimidos, como tem sido observado em pacientes com aids. Verificou-se descompensação cardíaca em um destes casos, com grave disfunção ventricular e arritmias sendo considerada a possibilidade de reagudização da doença de Chagas no miocárdio, uma vez que o xenodiagnóstico foi positivo. Face a gravidade foi tratado especificamente para o Trypanosoma cruzi com benznidazol, porém sem completar o tempo estipulado para este fim, vindo a falecer em consequiência de complicações da cardiopatia. A necropsia apresentou os estigmas habituais da cardiopatia chagásica crônica como miocardite fibrosante e redução do número de neurônios no tubo digestório, não sendo encontradas formas amastigotas do Trypanosoma cruzi em nenhum dos tecidos examinados. Assim, não ficou demonstrada a reagudização da doença de Chagas, mas sim evolução natural da cardiopatia chagásica crônica.
\end{abstract}

Palavras-chaves: Chagas. Tripanosomíase. Imunodeficiência adquirida. Reativação. Co-infecção.

\section{ABSTRACT}

Chagas disease is a type of parasitosis caused by the protozoan Trypanosoma cruzi, and it is transmitted by triatomine insects. This disease is found between the southern United States to Argentina and approximately 14 million people in Latin America are believed to be infected, predominantly with the chronic form of the disease. Reactivation of Chagas disease can occur among immunosuppressed patients, as has been observed among AIDS patients. In one such case, we observed cardiac decompensation with severe ventricular dysfunction and arrhythmias. This case was thought to be reactivation of Chagas disease in the myocardium, since the xenodiagnosis was positive. Specific treatment for Trypanosoma cruzi was administered, consisting of benznidazole, but the course of treatment was not completed because the patient died due to cardiopathic complications. The necropsy showed the usual stigmas of chronic Chagas cardiopathy, such as fibrosing myocarditis and a decreased number of neurons in the digestive system. There were no amastigote forms of Trypanosoma cruzi in any of the tissue samples studied. Therefore, reactivation of Chagas disease was not demonstrated but, rather, the natural evolution of chronic Chagas cardiopathy was demonstrated.

Key-words: Chagas. Trypanosomiasis. Acquired immunodeficiency. Reactivation. Coinfection.

A doença de Chagas ou tripanosomíase sul-americana é uma antropozoonose endêmica, ocorrendo desde o México até o sul da Argentina, causada por um protozoário flagelado, denominado Trypanosoma cruzi, transmitido ao homem e outros mamíferos por insetos hematófagos triatomínicos ${ }^{10}$. A forma de transmissão

1. Departamento de Clínica Médica, Faculdade de Ciências Médicas da Universidade Estadual de Campinas, Campinas, SP. 2. Disciplina de Parasitologia, Universidade Federal do Triângulo Mineiro, Uberaba, MG.

Endereço para correspondência: Dr. Eros Antonio de Almeida. Dept ${ }^{0}$ de Clínica Médica/FCM/UNICAMP. R. Alexander Fleming 40, Cidade Universitária Zeferino Vaz, 13083-970 Campinas, SP

Tel: 55 19 3521-7803; 5519 9257-9269; Fax: 55 19 3289-4107

e-mail: eros@fcm.unicamp.br

Recebido para publicação em 06/11/2008

Aceito em 20/03/2009 vetorial é a mais importante do ponto de vista de saúde pública, porém outras formas de transmissão existem, sendo a por transfusão sangüínea a segunda em importância epidemiológica. Após o contágio, ocorre uma fase aguda à qual se segue uma fase crônica, aproximadamente, depois de quatro meses da infecção e esta tem evolução variável em diferentes formas clínicas. É estimado que aproximadamente 14 milhões de pessoas estejam infectadas na América Latina e que 60 milhões estejam em risco de infecção, sendo que no Brasil, de 1,8 a 2,4 milhões de indivíduos devam estar na fase crônica da doença, 1/3 deles na forma cardíaca e digestiva, gerando alta morbi-mortalidade ${ }^{1}$.

Com o surgimento da infecção pelo vírus da imunodeficiência humana e o desenvolvimento da síndrome da imunodeficiência adquirida, passou a ser descrita a co-infecção desta com a doença de Chagas ${ }^{6} .0$ movimento migratório ocorrido no Brasil, 
das regiões rurais para as urbanas, a partir da década de 70, determinou a possibilidade, com maior frequiência, que indivíduos portadores da doença de Chagas se infectassem com o vírus da imunodeficiência, o qual é predominante em regiões urbanas. Fato semelhante tem ocorrido em outros países da América Latina 5 .

Uma situação de grave prognóstico que tem sido observada nesta co-infecção tem sido a meningoencefalite pelo Trypanosoma cruzi, caracterizando reagudização da doença de Chagas, uma vez que o sistema nervoso central não é acometido desta forma na fase crônica da doença ${ }^{8}$. Esta reagudização tem sido descrita, também, no coração ${ }^{14}$.

0 objetivo deste trabalho é relatar um caso de co-infecção Chagas/Aids em que havia evidências clínicas de reagudização da doença de Chagas no coração, o que não foi confirmada pela necropsia, gerando confusão diagnóstica.

\section{RELATO DO CASO}

Homem pardo, 37 anos, sabia ser portador da doença de Chagas, adquirida por provável via vetorial uma vez que nasceu e residiu em região endêmica para a tripanosomíase, não havia histórico de transfusão de sangue e desconhecia a doença na mãe. A doença de Chagas foi confirmada por sorologia para 0 Trypanosoma cruzi em mais de uma ocasião e por duas reações de métodos diferentes (imunofluorescência indireta para Chagas e fixação do complemento), mas não havia manifestações orgânicas desta doença. 0 doente adquiriu o vírus da imunodeficiência humana, por provável via de relação homossexual, sendo que o diangóstico foi efetuado seis anos antes do óbito por causa deste fator de risco. Sete meses antes do óbito, foi diagnosticada a síndrome da imunodeficiência, por ocasião de pneumonite por Pneumocystisjiroveci e, também toxoplasmose, tendo alcançado um bom controle clínico destas complicações.

Em vista de dificuldades técnicas de adesão aos serviços de acompanhamento, e também relativo ao próprio doente, não houve tempo hábil para a quantificação da carga viral, avaliação dos linfocitos, assim como de introdução de terapia anti-retroviral adequada. Neste período, passou a apresentar insuficiência cardíaca congestiva, sendo constatado grave miocardiopatia dilatada aos exames radiológicos do tórax e ecocardiograma, os quais demonstraram cardiomegalia e congestão pulmonar, dilatação do átrio e ventrículo esquerdo, fração de ejeção de $25 \%$, discinesia ventricular esquerda, além de insuficiência mitral moderada, porém sem lesão valvar. Exames eletrocardiográficos e Holter demonstraram bloqueios do ramo direito, divisional anterosuperior do ramo esquerdo e arritmias cardíacas, caracterizadas por extrassístoles supraventriculares e ventriculares, múltiplas, aos pares e em salvas. Cintilografia do miocárdio com Galium-60 demonstrou miocardite de intensidade moderada. Exame de xenodiagnóstico foi realizado por duas vezes, com 40 ninfas de $3^{\circ}$ a $5^{\circ}$ estágio do Triatoma infestans, sendo positivos apresentando apenas uma ninfa contaminada pelo Trypanosoma cruzi, por exame. 0 parasita não foi encontrado na pesquisa direta no sangue periférico. Apresentou sintomas e sinais neurológicos de comprometimento motor, com hemiplegia, lesão de sétimo par craniano e dislalia. A tomografia cerebral apresentou lesão hipodensa hemisferial esquerda periventricular, com efeito de massa sobre o corno anterior do ventrículo lateral esquerdo sem captação de contraste. Foi realizado exame do líquido cefaloraquidiano com pesquisa de Trypanosoma cruzi a qual foi negativa. Recebeu tratamento empírico para toxoplasmose, mas teve como hipótese diagnóstica diferencial isquemia cerebral devido à súbita instalação do processo neurológico. Com o quadro clínico cardíaco descompensado e parasitemia comprovada, embora de pequena intensidade, o diagnóstico de reagudização da doença de Chagas no coração foi aventada, sendo o doente medicado com benznidazol, $7 \mathrm{mg} / \mathrm{kg} /$ dia. 0 fármaco foi usado por apenas 22 dias, uma vez que se constatou aplasia de medula óssea, sendo creditado este achado a efeito adverso do benznidazol. Conduta adequada para a cardiopatia como uso de diuréticos, vasodilatadores, digitálicos, além de cuidados gerais intensivos foi instalada, no entanto, sem boa resposta clínica. 0 óbito ocorreu em consequiência da cardiopatia e suas complicações, tais como embolia pulmonar e cerebral, um mês e 11 dias do diagnóstico da provável reagudização.

Necropsia. Foram encontrados múltiplos infartos pulmonares e sequela de acidente vascular cerebral, sendo descartada qualquer possibilidade de meningoencefalite de qualquer etiologia. Havia grave cardiomegalia global, com trombose nos ventrículos e átrio direito. 0 exame histológico do miocárdio encontrou-se miocardite crônica fibrosante grave e diminuição dos neurônios do plexo intramural do tubo digestório. Não foram encontradas formas amastigotas do Trypanosoma cruzi no miocárdio e em nenhum outro tecido examinado. Não houve pesquisa do parasita por técnicas de imunohistoquímica ou biologia molecular.

\section{DISCUSSÃO}

A fase aguda da doença de Chagas tem se tornado rara após o controle das principais formas de transmissão, vetorial e transfusional. No entanto, tem sido verificada em indivíduos, sob tratamento quimioterápico para neoplasias malignas, transplantados de órgãos entre outras condições indutoras de imunossupressã $0^{7}$, caracterizando reagudizações da doença. Em portadores da síndrome da imunodeficiência adquirida, esta reagudização é relevante, uma vez que há mal prognóstico, uma vez que esta situação, habitualmente, compromete o sistema nervoso central ${ }^{6812}$. Assim, critérios têm sido propostos para se diagnosticar esta reagudização, uma vez que o tratamento específico com medicamentos antitripanosomicidas já demonstraram melhorar o prognóstico destes doentes ${ }^{11}$. Um dos critérios clínicos para a hipótese da reagudização tem sido a verificação da doença de Chagas em locais do organismo onde esta não incide com frequiência na fase crônica da doença, tal como o sistema nervoso central, sendo este local uma das principais expressões da reagudização da doença de Chagas na co-infecção com a imunodeficiência adquirida ${ }^{4}$. No entanto, o diagnóstico de confirmação é realizado pela presença do Trypanosoma cruzi no sangue periférico, nos fluidos orgânicos (líquor, 
derrames cavitários, etc) por métodos diretos de observação ou nos locais das lesões em indivíduos com a co-infecção. Tem sido proposto na literatura a verificação da parasitemia por métodos semiquantitativos $^{121314}$, sendo que a presença do parasita em mais do que $20 \%$ das ninfas observadas ao xenodiagnóstico determina parasitemia alta. Nos indivíduos com parasitemia alta, detectada por este método, a vigilância sobre a possibilidade de reagudização deverá ser maior. Quando menos do que $20 \%$ das ninfas encontramse contaminadas a parasitemia é considerada baixa e a evolução da doença de Chagas costuma ocorrer de forma natural ${ }^{13}$.

Em regiões alvo de lesões da doença de Chagas crônica, tais como o coração e o aparelho digestório, o diagnóstico da reagudização pode apresentar dificuldades, uma vez que a evolução natural da doença apresenta sinais e sintomas comuns a aqueles encontrados na reagudização. Estes seriam os quadros clínicos de descompensação em forma de insuficiência cardíaca, bloqueios e arritmias cardíacas tais como as verificadas neste caso pelo ecocardiograma, eletrocardiograma e Holter.

No caso apresentado, o diagnóstico clínico de reagudização foi aventado com base na descompensação da cardiopatia, com o doente apresentando insuficiência cardíaca de aparecimento recente, uma vez que sete meses atrás esta não ocorria. A parasitemia, confirmada pelo xenodiagnóstico por duas ocasiões, contribuiu para esta suspeita diagnóstica, embora o número de ninfas infectadas tenha sido pequeno, traduzindo baixa parasitemia ${ }^{9}$. A baixa parasitemia detectada ao xenodiagnóstico pode ser encontrada na fase crônica da doença de Chagas em indivíduos imunocompetentes e, isoladamente, não deve ser considerada como sinal de reagudização na co-infecção doença de Chagas/síndrome da imunodeficiência adquirida ${ }^{11}{ }^{13}$. No entanto, no caso apresentado achou-se prudente interpretar como tal, uma vez que, além da descompensação cardíaca e da comprovação da parasitemia, as demais condições do doente inerentes à imunodeficiência adquirida, como as infecções constatadas, indicavam a presença de imunossupressão. Embora o número reduzido de linfócitos TCD $4^{+}$, abaixo de 200cels $/ \mathrm{mm}^{3}$, e carga viral elevada sejam condições que demonstram grave imunossupressão e possam ser considerados como fatores preditivos para a reagudização da doença de Chagas na coinfecção com a imunodeficiência adquirida, nem sempre têm se demonstrado co-relação entre estes dados e a reagudização $0^{11}{ }^{12}$. No caso em discussão, não foi possível a verificação destes dois últimos critérios. No entanto, as evidências de infecções oportunísticas indicaram haver grave imunossupressão o que contribuiu para a impressão diagnóstica de reagudização da doença de Chagas.

A conduta clínica escolhida foi o tratamento específico para o Trypanosoma cruzi utilizando-se o medicamento benznidazol, uma vez que esta conduta tem beneficiado estes doentes, mudando a história natural da reagudização em co-infectados ${ }^{11}{ }^{12}$. Como não é infrequente com este tipo de medicamento ${ }^{2}$ ocorreu efeito adverso grave, culminando com a suspensão do benznidazol e o óbito do doente, sendo este interpretado como consequiência da reagudização da doença de Chagas no miocárdio. Neste sentido, a necropsia foi essencial para exclusão diagnóstica da reagudização uma vez que se detectaram apenas os estigmas da miocardite crônica e lesões digestórias habitualmente encontradas na forma crônica da doença de Chagas, não sendo observadas as formas intracelulares, amastigotas, do Trypanosoma cruzi em quantidades elevadas existentes nas formas reagudizadas da doença em imunossuprimidos. Como a necropsia foi feita dirigida pelo diagnóstico clínico de reagudização, esforços foram intensos na pesquisa dos parasitas intracelulares, não podendo, portanto, ser creditado à falta de atenção dos executores não se encontrar os parasitas. Outro fato a ser questionado para justificar o não encontro dos parasitas poderia ser a utilização do medicamento antitripanosomicida, benznidazol. Embora o tratamento preconizado com este medicamento para melhor eficácia seja de, no mínimo, 60 dias ${ }^{3}$, não se pode excluir com absoluta certeza que o uso deste por $1 / 3$ do período preconizado seja responsável por reduzir a parasitemia. Outras formas de confirmação da presença do parasita, tais como a imunohistoquímica e a biologia molecular por técnica da reação em cadeia da polimerase (PCR), não têm sido ainda apresentadas na literatura como adequadas ao diagnóstico da reagudização da doença de Chagas na co-infecção com a síndrome da imunodeficiência adquirida ${ }^{11}$.

Deste modo, concluímos que, neste caso, possa não ter ocorrido a reagudização da doença de Chagas no coração. 0 caso apresentado demonstra a dificuldade de se fazer este diagnóstico em indivíduos com a co-infecção Chagas/imunodeficiência adquirida, uma vez que pode se confundir a evolução natural da cardiopatia chagásica crônica com o quadro clínico da reagudização. Em vista da dificuldade diagnóstica de certeza nesta situação, parece justificável a utilização de medicamentos antitripanosomicidas em havendo suspeita diagnóstica da reagudização no miocárdio, como foi feito no caso apresentado.

\section{REFERÊNCIAS}

1. Akhavan D. Análise de custo-efetividade do programa de controle da doença de Chagas no Brasil. Relatório final. Organização Pan-Americana da Saúde, Brasília p.271, 2000 .

2. Castro JA, Mecca MM, Bartel LC. Toxic effects of drugs used to treat Chagas'disease (American trypanosomiasis). Human Experimental Toxicology 25:471-479, 2006 .

3. Consenso Brasileiro em Doença de Chagas. Revista da Sociedade Brasileira de Medicina Tropical 38 (supl III): 7-29, 2005.

4. Cordova E, Boschi A, Ambrosioni J, Cudos C, Corti M. Reactivation of Chagas' disease with central nervous involvement in HIV-infected patients in Argentina, 1992-2007. International Journal of Infectious Disease 12:587-592, 2008.

5. Corti M. AIDS and Chagas'disease. AIDS Patient Care and STDs 14: 581-588, 2000.

6. Del Castillo M, Mendoza G, Oviedo J. AIDS and Chagas'disease with central nervous system tumor-like lesion. The American Journal of Medicine 88:693-694, 1990.

7. Ferreira MS, Nishioka AS, Rocha A, Silva AM. Doença de Chagas e imunossupressão. In: Dias JCP, Coura JR (eds) Clínica e Terapêutica da Doença de Chagas, $1^{\mathrm{a}}$ edição, Fundação Osvaldo Cruz, p.365-379, 1997.

8. Santos EO, Reis Canela J, Gomes Monção HC, Guedes Roque MJ. Reactivation of Chagas'disease leading to the diagnosis of acquired immunodeficiency syndrome. Brazilian Journal of Infectious Disease 6: 317-321, 2002.

9. PereiraJB, Willcox HPP, Marcondes CB, Coura JR. Parasitemia em pacientes chagásicos crônicos avaliados pelo índice de triatomíneos infectados no xenodiagnóstico. Revista da Sociedade Brasileira de Medicina Tropical 22: 39-44, 1989. 
10. Rassi Jr A, Rassi A, Little WC. Chagas' heart disease. Clinical Cardiology 23:883-889, 2000.

11. Recommendations for diagnosis, treatment and follow-up of the Trypanosoma cruzi - human immunodeficiency virus co-infection. Thecnical Report of Health Ministery of Brazil 2006. Revista da Sociedade Brasileira de Medicina Tropical 39:392-341, 2006.

12. Sartori AM, Ibrahim KY, Nunes EVW, Braz LM, Oliveira Jr OC, Gakiya E, Lopes MH, Shikanai-Yasuda MA. Manifestations of Chagas'disease (American trypanosomiasis) in patients with HIV/AIDS. Annals of Tropical Medicine Parasitology 101: 31-50, 2007.
13. Sartori AMC, Eluf Neto J, Nunes EV, Braz LMA, Caiaffa-Filho HH, Oliveira Jr OC, Amato Neto V, Shikanai-Yassuda MA. Trypanosoma cruzi parasitemia in chronic Chagas' disease: comparison between Human Immunodeficiency Virus (HIV)-Positive and HIV-Negative patients. The Journal of Infectious Diseases $186: 872-875,2002$

14. Sartori AM, Shikanai-Yasuda MA, Amato Neto V, Lopes MH. Follow-up of 18 patients with human immunodeficiency vírus infection and chronic Chagas'disease with reactivation of Chagas'disease causing cardiac disease in three patients. Clinical of Infectious Disease 26:177-179, 1998. 
derrames cavitários, etc) por métodos diretos de observação ou nos locais das lesões em indivíduos com a co-infecção. Tem sido proposto na literatura a verificação da parasitemia por métodos semiquantitativos $^{121314}$, sendo que a presença do parasita em mais do que $20 \%$ das ninfas observadas ao xenodiagnóstico determina parasitemia alta. Nos indivíduos com parasitemia alta, detectada por este método, a vigilância sobre a possibilidade de reagudização deverá ser maior. Quando menos do que $20 \%$ das ninfas encontramse contaminadas a parasitemia é considerada baixa e a evolução da doença de Chagas costuma ocorrer de forma natural ${ }^{13}$.

Em regiões alvo de lesões da doença de Chagas crônica, tais como o coração e o aparelho digestório, o diagnóstico da reagudização pode apresentar dificuldades, uma vez que a evolução natural da doença apresenta sinais e sintomas comuns a aqueles encontrados na reagudização. Estes seriam os quadros clínicos de descompensação em forma de insuficiência cardíaca, bloqueios e arritmias cardíacas tais como as verificadas neste caso pelo ecocardiograma, eletrocardiograma e Holter.

No caso apresentado, o diagnóstico clínico de reagudização foi aventado com base na descompensação da cardiopatia, com o doente apresentando insuficiência cardíaca de aparecimento recente, uma vez que sete meses atrás esta não ocorria. A parasitemia, confirmada pelo xenodiagnóstico por duas ocasiões, contribuiu para esta suspeita diagnóstica, embora o número de ninfas infectadas tenha sido pequeno, traduzindo baixa parasitemia ${ }^{9}$. A baixa parasitemia detectada ao xenodiagnóstico pode ser encontrada na fase crônica da doença de Chagas em indivíduos imunocompetentes e, isoladamente, não deve ser considerada como sinal de reagudização na co-infecção doença de Chagas/síndrome da imunodeficiência adquirida ${ }^{11}{ }^{13}$. No entanto, no caso apresentado achou-se prudente interpretar como tal, uma vez que, além da descompensação cardíaca e da comprovação da parasitemia, as demais condições do doente inerentes à imunodeficiência adquirida, como as infecções constatadas, indicavam a presença de imunossupressão. Embora o número reduzido de linfócitos TCD $4^{+}$, abaixo de 200cels $/ \mathrm{mm}^{3}$, e carga viral elevada sejam condições que demonstram grave imunossupressão e possam ser considerados como fatores preditivos para a reagudização da doença de Chagas na coinfecção com a imunodeficiência adquirida, nem sempre têm se demonstrado co-relação entre estes dados e a reagudização $0^{11}{ }^{12}$. No caso em discussão, não foi possível a verificação destes dois últimos critérios. No entanto, as evidências de infecções oportunísticas indicaram haver grave imunossupressão o que contribuiu para a impressão diagnóstica de reagudização da doença de Chagas.

A conduta clínica escolhida foi o tratamento específico para o Trypanosoma cruzi utilizando-se o medicamento benznidazol, uma vez que esta conduta tem beneficiado estes doentes, mudando a história natural da reagudização em co-infectados ${ }^{11}{ }^{12}$. Como não é infrequente com este tipo de medicamento ${ }^{2}$ ocorreu efeito adverso grave, culminando com a suspensão do benznidazol e o óbito do doente, sendo este interpretado como consequiência da reagudização da doença de Chagas no miocárdio. Neste sentido, a necropsia foi essencial para exclusão diagnóstica da reagudização uma vez que se detectaram apenas os estigmas da miocardite crônica e lesões digestórias habitualmente encontradas na forma crônica da doença de Chagas, não sendo observadas as formas intracelulares, amastigotas, do Trypanosoma cruzi em quantidades elevadas existentes nas formas reagudizadas da doença em imunossuprimidos. Como a necropsia foi feita dirigida pelo diagnóstico clínico de reagudização, esforços foram intensos na pesquisa dos parasitas intracelulares, não podendo, portanto, ser creditado à falta de atenção dos executores não se encontrar os parasitas. Outro fato a ser questionado para justificar o não encontro dos parasitas poderia ser a utilização do medicamento antitripanosomicida, benznidazol. Embora o tratamento preconizado com este medicamento para melhor eficácia seja de, no mínimo, 60 dias ${ }^{3}$, não se pode excluir com absoluta certeza que o uso deste por $1 / 3$ do período preconizado seja responsável por reduzir a parasitemia. Outras formas de confirmação da presença do parasita, tais como a imunohistoquímica e a biologia molecular por técnica da reação em cadeia da polimerase (PCR), não têm sido ainda apresentadas na literatura como adequadas ao diagnóstico da reagudização da doença de Chagas na co-infecção com a síndrome da imunodeficiência adquirida ${ }^{11}$.

Deste modo, concluímos que, neste caso, possa não ter ocorrido a reagudização da doença de Chagas no coração. 0 caso apresentado demonstra a dificuldade de se fazer este diagnóstico em indivíduos com a co-infecção Chagas/imunodeficiência adquirida, uma vez que pode se confundir a evolução natural da cardiopatia chagásica crônica com o quadro clínico da reagudização. Em vista da dificuldade diagnóstica de certeza nesta situação, parece justificável a utilização de medicamentos antitripanosomicidas em havendo suspeita diagnóstica da reagudização no miocárdio, como foi feito no caso apresentado.

\section{REFERÊNCIAS}

1. Akhavan D. Análise de custo-efetividade do programa de controle da doença de Chagas no Brasil. Relatório final. Organização Pan-Americana da Saúde, Brasília p.271, 2000 .

2. Castro JA, Mecca MM, Bartel LC. Toxic effects of drugs used to treat Chagas'disease (American trypanosomiasis). Human Experimental Toxicology 25:471-479, 2006 .

3. Consenso Brasileiro em Doença de Chagas. Revista da Sociedade Brasileira de Medicina Tropical 38 (supl III): 7-29, 2005.

4. Cordova E, Boschi A, Ambrosioni J, Cudos C, Corti M. Reactivation of Chagas' disease with central nervous involvement in HIV-infected patients in Argentina, 1992-2007. International Journal of Infectious Disease 12:587-592, 2008.

5. Corti M. AIDS and Chagas'disease. AIDS Patient Care and STDs 14: 581-588, 2000.

6. Del Castillo M, Mendoza G, Oviedo J. AIDS and Chagas'disease with central nervous system tumor-like lesion. The American Journal of Medicine 88:693-694, 1990.

7. Ferreira MS, Nishioka AS, Rocha A, Silva AM. Doença de Chagas e imunossupressão. In: Dias JCP, Coura JR (eds) Clínica e Terapêutica da Doença de Chagas, $1^{\mathrm{a}}$ edição, Fundação Osvaldo Cruz, p.365-379, 1997.

8. Santos EO, Reis Canela J, Gomes Monção HC, Guedes Roque MJ. Reactivation of Chagas'disease leading to the diagnosis of acquired immunodeficiency syndrome. Brazilian Journal of Infectious Disease 6: 317-321, 2002.

9. PereiraJB, Willcox HPP, Marcondes CB, Coura JR. Parasitemia em pacientes chagásicos crônicos avaliados pelo índice de triatomíneos infectados no xenodiagnóstico. Revista da Sociedade Brasileira de Medicina Tropical 22: 39-44, 1989. 
10. Rassi Jr A, Rassi A, Little WC. Chagas' heart disease. Clinical Cardiology 23:883-889, 2000.

11. Recommendations for diagnosis, treatment and follow-up of the Trypanosoma cruzi - human immunodeficiency virus co-infection. Thecnical Report of Health Ministery of Brazil 2006. Revista da Sociedade Brasileira de Medicina Tropical 39:392-341, 2006.

12. Sartori AM, Ibrahim KY, Nunes EVW, Braz LM, Oliveira Jr OC, Gakiya E, Lopes MH, Shikanai-Yasuda MA. Manifestations of Chagas'disease (American trypanosomiasis) in patients with HIV/AIDS. Annals of Tropical Medicine Parasitology 101: 31-50, 2007.
13. Sartori AMC, Eluf Neto J, Nunes EV, Braz LMA, Caiaffa-Filho HH, Oliveira Jr OC, Amato Neto V, Shikanai-Yassuda MA. Trypanosoma cruzi parasitemia in chronic Chagas' disease: comparison between Human Immunodeficiency Virus (HIV)-Positive and HIV-Negative patients. The Journal of Infectious Diseases $186: 872-875,2002$

14. Sartori AM, Shikanai-Yasuda MA, Amato Neto V, Lopes MH. Follow-up of 18 patients with human immunodeficiency vírus infection and chronic Chagas'disease with reactivation of Chagas'disease causing cardiac disease in three patients. Clinical of Infectious Disease 26:177-179, 1998. 